\title{
Erdbeeranbau für die Industriebelieferung
}

\author{
von Gerhard Mellenthin, Hamburg-Volksdorf
}

Ein erheblicher Teil der jährlichen Erdbeerernte wird von der verarbeitenden Industrie aufgenommen. In Deutschland ist der Anteil der Industrie am Verbrauch von Erdbeeren in den letzten Jahren ständig gestiegen und kann heute auf etwa $40-50 \%$ des Marktangebotes geschätzt werden. Nimmt man also Eigenerzeugung und Importe zusammen, so entspricht das einer Menge von etwa $16.000-20.000 \mathrm{t}$ jährlich. Die genaue Menge läBt sich nicht nachweisen. Mit steigendem Lebensstandard ist $\mathrm{zu}$ erwarten, daB der Anteil der verarbeiteten Frucht am Gesamtkonsum weiter ansteigt $(1,2,3)$.

Der verstärkten Nachfrage von seiten der Industrie muß sich der Anbau anpassen. Bisher ist das im deutschen Anbau zweifellos nicht im möglichen Umfange geschehen. Die Folge ist, $\mathrm{da} B$ die Industrie in zunehmendem Maße ihren Bedarf im Ausland deckt. Nur in dem nach dem. Kriege neu entstandenen norddeutschen Anbaugebiet zwischen Stade, Hamburg und Lübedk kann man heute von einem planmäßigen Industrieanbau sprechen, obwohl auch hier die Zusammenarbeit zwischen Anbauer und Fabrik noch verbesserungsfähig ist.

Ein Erdbeeranbau für die Industriebelieferung unterscheidet sich in wesentlichen Punkten von einem Anbau für den Frischmarkt. Bevor die erste Fläche bepflanzt wird, müssen die Absatzmöglichkeiten und die näheren Bedingungen geklärt sein Industrieanbau verlangt also, wenn er reibungslos funktionieren soll, eine eingehende Vorplanung. Es empfiehlt sich dafür die Anforderungen, die die Industrie stellt, im einzelnen kennenzulernen. Wenn es audh je nach der speziellen Art der Verarbeitung bei den Fabriken Unterschiede gibt, so stehen doch ganz allgemein folgende Wünsche im Vordergrund:
1. Für die Verarbeitung geeignete Sorten
2. Kurzer Transportweg
3. Geschlossene Anlieferung einer einheitlichen Ware
4. Hohe Qualität
5. Niedriger Preis
6. Sicherung des jährlichen Bedarfs

Ein erfolgreicher Industrieanbau muß die Konsequenzen aus diesen Forderungen ziehen.

\section{Sorten}

Es ist kein Zufall, daß die Entstehung des norddeutschen Anbaugebietes mit der Einführung der Sorte SENGA SENGANA zusammenfällt. Sie gilt heute nicht nur in Deutschland, sondern auch in Holland und den meisten anderen europäischen Ländern als Standardsorte für die industrielle Verarbeitung.

\section{Standort}

Der Industrieanbau sollte in einem Umkreis von etwa 50 bis $100 \mathrm{~km}$ von der Fabrik lokalisiert sein. Allen modernen Verkehrsmitteln zum Trotz gelangen die in der Nähe der Fabrik erzeugten Beeren ungleich frischer zur Verarbeitung als Früchte, die einen längeren Transport hinter sich haben. Wenn Anbauer und Fabrik gut harmonieren, ist es zu erreichen, daß sogar mehrmals täglich die frisch gepflückten Beeren von der Plantage abgeholt werden und nur wenige Stunden später verarbeitet sind. Die Fabriknähe bedeutet darüber hinaus eine nennenswerte Verminderung der Transportkosten.

\section{Grobe Flächen}

Die Fabriken benötigen für eine rentable Ausnutzung ihrer Máschinen täglich gewisse Mindestmengen an Früchten. Dementsprechend müssen entweder die Erdbeerflächen je Betrieb so groß sein, daß lohnende Tagesmengen bereitgestellt werden können oder aber in einem geschlossenen Anbaugebiet werden in einer Zentrale, etwa in einer Genossenschaft, die kleinen Mengen der einzelnen Betriebe zu einem Großangebot zusammengefaßt. In Norddeutschland dominiert der Aribau in landwirtschaftlichén Betrieben. Die Erdbeerfläche je Betrieb ist relativ groß und beträgt in der Regel mehrere Hektar. Die Entwidklung zur größeren Erdbeerfläche je Betrieb ist noch nicht abgeschlossen. Es hat sich in den landwirtschaftlichen Betrieben gezeigt, daß eine Sonderkultur wie die Erdbeere eine Spezialisierung des ganzen Betriebes und damit größere Flächen verlangt. Ein Landwirt, der diesen Weg nicht gehen will, wird den Erdbeeranbau nach kurzer Zeit wieder aufgeben; weil er in seinem Betrieb stört.

Der Großanbau sieht seinen Vorteil in der billigeren Bewirtschaftung der Flächen. So werden eine Reihe von Rationalisierungsmaßnahmen $a b$ einer gewissen Mindestfläche überhaupt erst möglich und voll wirksam. Das trifft insbesondere auf den Einsatz von Maschinen, aber auch auf die Akkordbezahlung der Handarbeiten zu. Nicht die Fläche, sondern die Arbeitskraft ist heute der begrenzende Faktor. Obwohl man natürlich andererseits für größere Flächen mehr Arbeitskräfte benötigt, so liegt darin doch kein Widerspruch, weil es darauf ankommt, auf der großen Fläche eine höhere Leistung je Arbeitskraft zu erzielen. Die obere Grenze der Flächengröße wird in der Regel von der örtlichen Möglichkeit bestimmt, eine genügende Anzahl Pflücker zu beschaffen. Der Anbauer muß also anstreben, nicht nur hohe Erträge je Fläche zu erzielen, sondern auch diese Erträge mit dem geringstmöglichen Arbeitsaufwand zu erreichen. Im Hinblick auf die Preisgestaltung bei Industriebelieferung kommt diesem Gesichtspunkt eine besondere Bedeutung zu.

\section{Qualität}

Als überholt muB heute die Methode angesehen werden, in einem Betrieb den Anbau für den Markt und für die Industrie zu mischen. Auch die alte Praxis, die guten Qualitäten für den Markt und die abfallende Ware für die Fabrik zu sortieren, ist nicht mehr vertretbar. Die Industrie steht heute mit ihren Produkten genauso im Qualitätswettbewerb wie er auf dem Frischmarkt schon immer vorhanden war. Sie muß also ihrerseits bei der Beschaffung der Rohware strengste Qualitätsmaßstäbe anlegen. Die Ernte für die Fabrik verlangt deshalb eine genauso sorgfältige Behandlung der Beeren wie für den Frischmarkt. Es gibt sogar Angebote aus dem Ausland, bei denen die Beeren nach Größe sortiert sind, um den unterschiedlichen Verarbeitungszwecken noch besser gerecht zu werden.

$\mathrm{DaB}$ Industriebeeren entkelcht gepflückt werden, ist heute eine selbstyerständliche Voraussetzung, bei der Sorte SENGA SENGANA auch ohne Schwierigkeiten möglich (4). Da entkelchte Beeren auf dem Frischmarkt nicht abzusetzen sind, ist schon deshalb eine Trennung von Industrie- und Frischmarktflächen erforderlich.

Preis

Um einen gerechten Preis pflegen im Verhältnis zwischen Anbauer und Fabrik die größten Differenzen zu entstehen. In den meisten Fällen werden die Fabriken ihren Bedarf täglich partienweise kaufen. Hierfür wird ein Preis ausgehandelt, der sich am Frischmarktpreis und am Importpreis orientiert. Da die Industrie den Verkaufspreis für ihr Fertigprodukt in der Regel als Einheitspreis für das ganze Jahr kalkuliert, ist sie neuerdings stärker bestrébt, auch für die Rohware einen Durch- 
schnittspreis für die ganze Erntedauer zu vereinbaren. Eine solche Regelung muß für den Anbauer nicht ungünstig sein: verlangt von ihm aber eine grundsätzlich andere Einstelling als bei einem Verkauf über den Markt.

Mit einem Festpreis, der vor Beginn der Ernte vereinbart wird, entfällt die Chance, bei guter Marktlage hohe Preise zu erzielen, es entfällt aber auch das Risiko eines zu niedrigen Preises bei Marktzusammenbrüchen. Auch Festpreisvereinbarungen richten sich nach Angebot und Nachfrage. Vorhandene Anbaufläche, Stand der Kulturen, erwartete Erntemengen, Räumung der Läger in den Fabriken, erwarteter Bedarf dés Konsums und damit beabsichtigter Produktionsumfang der Industrie spielen dabei eine Rolle. Für den Anbauer liegt bei einer Lieferung an die Industrie der Vorteil weniger in einem absolut hohen Erlös als vielmehr in einer relativ. risikofreien Sicherung einer ausreichenden Rente. Er sollte übertrieben hohe Preisforderungen vermeiden, wenn sie nicht marktgerecht sind, weil er damit die Fabrik zwingt, zur Konkurrenz, d. h. zum Auslandslieferanten abzuwandern. Andererseits müssen wir auch von der Industrie eine reelle Einstellung zu einem vernünftigen Preis erwarten, wenn sie die Vorteile des einheimischen Anbaues ausnutzen will.

\section{Anbauverträge}

Der Industrieanbau kann auf die Vermittlerposition des Marktes verzichten. Bewährt haben sich dagegen die Genossenschaften, vornehmlich in ihrer Funktion der Sammlung von Kleinpartien. Es gibt auch das Beispiel der Kommissionsgeschäfte, die eine, echte und sinnvolle Mittlerrolle zwischen Anbauer und Industrie ausüben.

Besonders typisch für den Industrieanbau ist aber der $\mathrm{Ab}$ schluß von Anbau- und Lieferungsverträgen zwischen Fabriken und Anbauer direkt. Solche Verträge werden im einzelnen sehr unterschiedlich gehandhabt.

Bei dem Anbau- und Liefervertrag ohne Preisfestsetzung wird die Ablieferung und Abnahme einer geschätzten Erntemenge von einer bestimmten Fläche vereinbart. Der Preis wird entweder in der Saison täglich entsprechend der Marktlage festgesetzt, oder er wird kurz vor Beginn der Ernte als Einheitspreis für die gesamten Ablieferungen ausgehandelt.' Es gibt auch Vereinbarungen über einen festen Mindestpreis und einen festen Höchstpreis, zwischen denen in der Ernte der jeweilige Tagespreis nach dem Marktpreis ermittelt wird.
Bei dem Anbau- und Liefervertrag mit Preisfestsetzung wird von vornherein ein bestimmter Einheitspreis festgelegt. Häufig laufen solche Verträge über mehrere. Jahre. Es gibt außerdem verschiedene Fabriken, die sich an der Vorfinanzierung der Pflanzenkäufe des Anbauers beteiligen. Die Beträge'werden dann mit den Beerenlieferungen verrechnet.

Man kennt weiterhin - besonders in Skandinavien - Vertäge, die eine wesentlich engere Bindung des Erzeugers an die Fabrik' bedeuten. Sie sehen vor, daB der Anbauier das Land stellt und die Pflege durchführt. Das Pflanzgut wird von der Fabrik gestellt, und häufig wird auch die Ernte von der Fabrik aus durchgeführt. Der Anbauer erhält praktisch, keinen regulären Preis mehr, sondern eine Art Rente für sein Land und ein Entgelt für seine Arbeitsleistung. Diese Verträge lassen ihm zwar nur noch sehr geringe. Freiheit, nehmen ihm aber auch weitgehend das Risiko ab.

Verträge sollten so ausgearbeitet sein, $\mathrm{daB}$ sie spätere Streitigkeiten ausschiließen. Es muß also geregelt sein, wer die Körbe stellt und die Košten hierfür trägt, wer den Transport vom Feld zur Fabrik durchführt, wann die Abholung erfolgt und wer die Kosten trägt. Es. muß auch geregelt sein, nach welchen Gesîchtspunkten Réklamationen erfolgen können. Es ist-leider eine bekannte Tatsache, dáß bei einer reichlichen Ernte die Fabrik leicht geneigt ist, ungenügende Qualität zu reklamieren, und daß bei einer knappen Ernte der Anbauer in Versuchung gerät, einen Teil der für die Fabrik vorgesehenen Ernte auf dem Frischmarkt zu höherem Preis zu verkaufen.

Wenn beide Partner jedoch ein dauerhaftes, harmonisches Verhältnis anstreben, wird es sich von selbst ergeben, daß alle strittigen Punkte mit. Vernunft und Sorgfalt rechtzeitig jährlich besprochen und geklärt werden. Die Position der Anbauer kann dabei durch einen Zusammenschluß zu einem Anbauerring oder zu einer Absatzgenossenschaft wesentlich gestärkt werden.

Literaturverzeichnis:

1. Storck, H. und Massante, St. 1960: Der westdeutsche Markt für Erdbeeren; in: Erwerbsobstbau 2. Jg., H. 5, S. 81-84.

2. Lappe, K. H. 1961: Die Entwlcklung des Frdbeermarktes im letzten Jahrzehnt; in: Deutsche Gartenbauwirtschaft 9. Jg., H. 7, S. 133-135.

3. Mellenthin, G. 1962: Erdbeergroßanbau zur Industriebelieferung; Vortrag XVI. Internationaler Gartenbaukongreß.

4. Jordan, Chr. 1961: Erdbeeren - entkelcht gepfluckt; in: Deutsche Gartenbauwirtschaft 9. Jg., H. 7, S. 147. 